\title{
ON THE HEART OF HYPERMODULES
}

\author{
S. M. ANVARIYEH and B. DAVVAZ
}

\begin{abstract}
This paper presents some types of hypermodules, associated to an arbitrary hypermodule, studying properties and characterizing their hearts. Also, we establish a few results concerning the sequence of heart, which can be associated to a hypermodule, in connection with subhypermodules generated by a non-empty set, by a union of subhypermodules or by the intersection of subhypermodules. Finally, we study several properties of 1-hypermodules.
\end{abstract}

\section{Introduction}

A hypergroupoid $(H, \circ)$ is a non-empty set $H$ with a hyperoperation $\circ$ defined on $H$, that is, a mapping of $H \times H$ into the family of non-empty subsets of $H$. If $A, B$ are non-empty subsets of $H$ then $A \circ B$ is given by $A \circ B=$ $\bigcup\{x \circ y \mid x \in A, y \in B\} . x \circ A$ is used for $\{x\} \circ A$ and $A \circ x$ for $A \circ\{x\}$. A hypergroupoid $(H, \circ)$ is called a hypergroup in the sense of Marty if for all $x, y, z \in H$ the following two conditions hold: (i) $x \circ(y \circ z)=(x \circ y) \circ z$, (ii) $x \circ H=H \circ x=H$. If $(H, \circ)$ satisfies only the first axiom, then it is called a semi-hypergroup. An exhaustive review updated to 1992 of hypergroup theory appears in [2]. A recent book [3] contains a wealth of applications.

Let $H$ be a hypergroup and $\rho$ an equivalence relation on $H$. Let $\rho(a)$ be the equivalence class of $a$ with respect to $\rho$ and let $H / \rho=\{\rho(a) \mid a \in H\}$. A hyperoperation $\otimes$ is defined on $H / \rho$ by $\rho(a) \otimes \rho(b)=\{\rho(x) \mid x \in$ $\rho(a) \circ \rho(b)\}$. If $\rho$ is strongly regular, then it readily follows that $\rho(a) \otimes \rho(b)=$ $\{\rho(x) \mid x \in a \circ b\}$. It is well known for $\rho$ strongly regular that $(H / \rho, \otimes)$ is a group (see Theorem 31 in [2]), that is $\rho(a) \otimes \rho(b)=\rho(c)$ for all $c \in a \circ b$.

A hyperring is a multi-valued system $(R,+, \circ)$ which satisfies the ring-like axioms in the following way: $(R,+)$ is a hypergroup in the sense of Marty, $(R, \circ)$ is a semi-hypergroup and the multiplication is distributive with respect to the hyperoperation + . Let $R$ be a hyperring. We recall the relation $\Gamma$ defined as follows: $x \Gamma y \Longleftrightarrow \exists n \in \mathrm{N}, \exists\left(k_{1}, \ldots, k_{n}\right) \in \mathrm{N}^{n}$, and $\left[\exists\left(x_{i 1}, \ldots, x_{i k_{i}}\right) \in\right.$ 
$\left.R^{k_{i}},(i=1, \ldots, n)\right]$ such that

$$
x, y \in \sum_{i=1}^{n}\left(\prod_{j=1}^{k_{i}} x_{i j}\right) .
$$

The relation $\Gamma$ is reflexive and symmetric. Let $\Gamma^{*}$ be the transitive closure of $\Gamma$, then $\Gamma^{*}$ is a strongly regular relation both on $(R,+)$ and $(R, \cdot)$, and the quotient $R / \Gamma^{*}$ is a ring [5], [6].

Let $(M,+)$ be a hypergroup and $(R,+, \cdot)$ be a hyperring. According to [7] $M$ is said to be a hypermodule over $R$ if there exists

$$
\cdot: R \times M \rightarrow \wp^{*}(M) ; \quad(a, m) \mapsto a \cdot m
$$

such that for all $a, b \in R$ and $m_{1}, m_{2}, m \in M$, we have

(1) $a \cdot\left(m_{1}+m_{2}\right)=a \cdot m_{1}+a \cdot m_{2}$,

(2) $(a+b) \cdot m=(a \cdot m)+(b \cdot m)$,

(3) $(a \cdot b) \cdot m=a \cdot(b \cdot m)$.

Let $M$ be an $R$-hypermodule, $N_{1}$ and $N_{2}$ two subhypermodules of $M$; we say that $N_{2}$ is $N_{1}$-conjugable if $N_{2}$ as a subhypergroup is conjugable, and an $R$-hypermodule $M$ is regular if $M$ as a hypergroup is regular.

Let $R$ be a hyperring and $M$ be a hypermodule over $R$. Let $x, y \in M$, the relation $\epsilon$ on $M$ defined as follows [4]:

$$
\begin{gathered}
x \in y \Leftrightarrow x, y \in \sum_{i=1}^{n} m_{i}^{\prime} ; \quad m_{i}^{\prime}=m_{i} \quad \text { or } \quad m_{i}^{\prime}=\sum_{j=1}^{n_{i}}\left(\prod_{k=1}^{k_{i j}} x_{i j k}\right) z_{i}, \\
m_{i} \in M, \quad x_{i j k} \in R, \quad z_{i} \in M .
\end{gathered}
$$

If $M$ is an $R$-hypermodule, then we set

$$
\epsilon_{0}=\{(m, m) \mid m \in M\}
$$

and for every integer $n \geq 1, \epsilon_{n}$ is the relation defined as follows:

$$
x \epsilon_{n} y \Leftrightarrow x, y \in \sum_{i=1}^{n} m_{i}^{\prime} .
$$

Obviously, for every $n \geq 0$, the relation $\epsilon_{n}$ is symmetric, and the relation $\epsilon=\bigcup_{n \geq 0} \epsilon_{n}$ is reflexive and symmetric.

The fundamental relation $\epsilon^{*}$ on $M$ can be defined as the smallest equivalence relation such that the quotient $M / \epsilon^{*}$ is a module over the corresponding fundamental ring $R / \Gamma^{*}$ such that $M / \epsilon^{*}$ as a group is not abelian. 
Let $M$ and $N$ be $R$-hypermodules. A function $f: M \rightarrow N$ is called an $R$-homomorphism, if for every $(x, y) \in M^{2}$ and $r \in R$

$$
f(x+y)=f(x)+f(y) \quad \text { and } \quad f(r \cdot x)=r \cdot f(x) .
$$

If $H$ is an $R$-module and $f: M \rightarrow H$ is an $R$-homomorphism, we let $\operatorname{Ker} f=$ $\left\{m \in M \mid f(m)=0_{H}\right\}$. Moreover, the canonical projection $\phi_{M}: M \rightarrow M / \epsilon^{*}$ by $\phi_{M}(m)=\epsilon^{*}(m)$, is an $R$-homomorphism and $\omega(M):=\operatorname{Ker} \phi_{M}=\{m \in$ $\left.M \mid \phi_{M}(m)=0_{M / \epsilon^{*}}\right\}$.

If $M$ is a hypermodule and $\rho \subseteq M \times M$ is an equivalence relation then for all pairs $(A, B)$ of non-empty subsets of $M$, we set $A \overline{\bar{\rho}} B$ if and only if $a \rho b$ for all $a \in A$ and $b \in B$. The relation $\rho$ is said to be strongly regular to the right if $x \rho y$ implies $x \circ a \overline{\bar{\rho}} y \circ a$ and $x . r \overline{\bar{\rho}} y . r$ for all $x, y, a \in M$ and $r \in R$. Analogously, we can define strongly regular to the left. Moreover $\rho$ is called strongly regular if it is strongly regular to the right and to the left.

THEOREM 1.1. Let $M$ be an R-hypermodule and $\rho$ be a strongly regular relation on $M$. Then $(M / \rho, \oplus)$ is an $R$-hypermodule if and only if for every $x \in M$ and $r \in R, r \cdot \rho(x)=\rho(r \cdot x)$.

Proof. Since $\rho$ is strongly regular, the scalar hyperoperation $r \cdot \rho(x):=$ $\rho(r \cdot x)$ is well defined. Since $M$ is an $R$-hypermodule, the properties of $M$ as an $R$-hypermodule, guarantee that the hypergroup $M / \rho$ is an $R$-hypermodule.

\section{Heart of a hypermodule}

In the following $m_{i}^{\prime}, z_{i}^{\prime}$ and $y_{i}^{\prime}$ are the notations in the definition of $\epsilon$.

Let $M$ be an $R$-hypermodule and $A$ be a non-empty subset of $M$. Then the intersection of the subsets of $M$ which are complete and contain $A$ is called the complete closure of $A$ in $M$; it will be denoted $C(A)$. If $K_{1}(A)=A$, and

$$
K_{n+1}(A)=\left\{x \in M \mid \exists p \in \mathrm{N}, \exists\left(m_{1}^{\prime}, m_{2}^{\prime}, \ldots, m_{p}^{\prime}\right): x \in \sum_{i=1}^{p} m_{i}^{\prime} \cap K_{n} \neq \emptyset\right\},
$$

then $K(A)=C(A)$, and the relation $x K y \Leftrightarrow x \in C(\{y\})$ is an equivalence. Also, for every $x, y \in M$, we have $x K y \Leftrightarrow x \epsilon^{*} y$. Furthermore, if $B$ is a non-empty subset of $M$, we have $C(B)=\bigcup_{b \in B} C(b)$, where $C(b)=C(\{b\})$ [1].

Theorem 2.1. Let $M$ be an R-hypermodule, $\phi_{M}: M \rightarrow M / K$ the canonical projection. If $N$ is a hypermodule with ordinary group and $f: M \rightarrow N$ is an R-homomorphism, then there exists $g: M / K \rightarrow N$ such that $g \phi_{M}=f$.

Proof. It is enough to observe for every $x \in M$ that $g \phi_{M}(x)=f(x)$. First, $g$ is well defined: in fact $\phi_{M}(x)=\phi_{M}(y) \Rightarrow x K y$. Since $N$ is a hypermodule 
(with ordinary group), it follows that $f(x)=f(y)$. Furthermore, $g$ is an $R$-homomorphism because for every $x, y \in M$, and $u \in x+y$, we have $g\left(\phi_{M}(x)+\phi_{M}(y)\right)=g \phi_{M}(x+y)=g \phi_{M}(u)=f(u)=f(x+y)=$ $f(x)+f(y)=g \phi_{M}(x)+g \phi_{M}(y)$. Also, for every $r \in R$, and $v \in r . x$ we have $g\left(\phi_{M}(r \cdot x)\right)=g\left(\phi_{M}(v)\right)=f(v)=f(r \cdot x)=r \cdot f(x)=r \cdot\left(g \phi_{M}(x)\right)$.

Theorem 2.2. Let $f: M \rightarrow M^{\prime}$ be an $R$-homomorphism, then:

(1) $\forall x \in M, f(C(x)) \subseteq C(f(x))$.

(2) $f$ determines an $R$-homomorphism $f^{*}: M / K \rightarrow M^{\prime} / K^{\prime}$ defined by $f^{*}\left(\phi_{M}(x)\right)=\phi_{M^{\prime}}(f(x))$.

Proof. (1) It is sufficient to observe that for every $n \in \mathrm{N}$, the implication $x \epsilon_{n} y \Rightarrow f(x) \epsilon_{n} f(x)$ is valid.

(2) $f^{*}$ is well defined, in fact $\phi_{M}(x)=\phi_{M}(y)$, i.e., $x K y$ implies by (1) $f(x) K f(y)$, hence $f^{*}\left(\phi_{M}(x)\right)=f^{*}\left(\phi_{M}(y)\right)$. Clearly, $f^{*}$ is an $R$-homomorphism, because for every $u \in x+y, f^{*}\left(\phi_{M}(x)+\phi_{M}(y)\right)=f^{*}\left(\phi_{M}(f(x+\right.$ $y)))=\phi_{M^{\prime}}(f(u))=\phi_{M^{\prime}}(f(u))=\phi_{M^{\prime}}(f(x)+f(y))=\phi_{M^{\prime}}(f(x))+$ $\phi_{M^{\prime}}(f(y))=f^{*}\left(\phi_{M}(x)\right)+f^{*}\left(\phi_{M}(y)\right)$, and for every $r \in R$ and $v \in r . x$, we have $f^{*}\left(\phi_{M}(r \cdot x)\right)=\phi_{M^{\prime}}(f(v))=\phi_{M^{\prime}}(f(r \cdot x))=r \cdot \phi_{M}(f(x))=$ $r \cdot f^{*}\left(\phi_{M}(x)\right)$.

LEMMA 2.3. For every non empty subset $H$ of an $R$-hypermodule $M$, we have

(1) $\phi_{M}^{-1}\left(\phi_{M}(H)\right)=\omega(M)+H=H+\omega(M)$.

(2) If $H$ is a complete part of $M$, then $\phi_{M}^{-1}\left(\phi_{M}(H)\right)=H$.

Proof. (1) For every $x \in \omega(M)+H$, there exists a pair $(a, b) \in \omega(M) \times H$ such that $x \in a+b$, so $\phi_{M}(x) \subseteq \phi_{M}(a)+\phi_{M}(b)=0_{M / \epsilon^{*}}+\phi_{M}(b)=\phi_{M}(b)$. Therefore $x \in \phi_{M}^{-1}\left(\phi_{M}(b)\right) \subseteq \bar{\phi}_{M}^{-1}\left(\phi_{M}(H)\right)$.

Conversely, for every $x \in \phi_{M}^{-1}\left(\phi_{M}(H)\right)$, there exists an element $b \in H$ such that $\phi_{M}(x)=\phi_{M}(b)$. By the reproducibility there exists $a \in M$ such that $x \in a+b$, so $\phi_{M}(b)=\phi_{M}(x)=\phi_{M}(a)+\phi_{M}(b)$, hence $\phi_{M}(a)=0_{M / \epsilon^{*}}$ and $a \in \phi_{M}^{-1}\left(0_{M / \epsilon^{*}}\right)=\omega(M)$. Therefore, $x \in a+b \subseteq \omega(M)+H$. This proves that $\phi_{M}^{-1}\left(\phi_{M}(M)\right)=\omega(M)+H$. In the same way, it is possible to prove that $\phi_{M}^{-1}\left(\phi_{M}(H)\right)=H+\omega(M)$.

(2) It is obvious that $H \subseteq \phi_{M}^{-1}\left(\phi_{M}(H)\right)$. Moreover, if $x \in \phi_{M}^{-1}\left(\phi_{M}(H)\right)$, then there exists an element $b \in H$ such that $\phi_{M}(x)=\phi_{M}(b)$. Hence $x \in$ $\epsilon^{*}(x)=\epsilon^{*}(b) \subseteq H$ and $\phi_{M}^{-1}\left(\phi_{M}(H)\right) \subseteq H$.

Lemma 2.4. Let $M$ be an $R$-hypermodule. Then $\omega(M)$ is the intersection of all $R$-subhypermodules of $M$ that are complete parts. 
Proof. By Lemma 2.3, we have $\omega(M)+\omega(M)=\omega(M)$ as a hypermodule. Let $A \in \bigcap M_{i}$, where every $M_{i}$ is a complete part subhypergroup of $M$. Then $A+\omega(M)=A$. Also, $A$ is an invertible subhypermodule of $M$, hence

$\forall(a, x) \in A \times \omega(M), \exists b \in A: a \in b+x \Rightarrow a \in A+x \Rightarrow x \in A+a=A$.

Therefore $\omega(M) \subseteq A$.

THeOREm 2.5. Let $M$ be an R-hypermodule and $B$ the union of summations of finite numbers of $\sum_{i=1}^{n} m_{i}^{\prime}$, containing at least one right and at least one left identity and be scalar multiplicatively closed. Then $B=\omega(M)$.

Proof. We set $E_{l}\left(E_{r}\right)$ equal to the set of left (right) identities and $T=$ $\left\{P \in B \mid P \cap E_{l} \neq \emptyset, P \cap E_{r} \neq \emptyset\right\}$. Furthermore, for every $x \in M$, we denote by $i_{l}(x)\left(i_{r}(x)\right)$ the set of left (right) inverses of $x$. First, we prove that for every $a \in B, i_{l}(a) \subseteq B \supseteq i_{r}(a)$. Let $a \in B$, then there exists a $\sum_{i=1}^{n} m_{i}^{\prime}=P \in T$ such that $a \in P$. If $a^{\prime} \in i_{l}(a)$, then $e^{\prime} \in E_{l}$ exists such that $e^{\prime} \in a^{\prime}+a$; if $a^{\prime \prime} \in i_{l}(a)$, then $e^{\prime \prime} \in E_{r}$ exists such that $e^{\prime \prime} \in a+a^{\prime \prime}$. We now consider the $P_{1}=a^{\prime}+\sum_{i=1}^{n} m_{i}^{\prime}+a+a^{\prime \prime}$, we have $P_{1} \subseteq T$, in fact $\left\{e^{\prime}, e^{\prime \prime}\right\} \subseteq e^{\prime}+e^{\prime \prime} \subseteq a^{\prime}+a+a+a^{\prime \prime} \subseteq P_{1}$. Furthermore, $\left\{a^{\prime}, a^{\prime \prime}\right\} \subseteq P_{1}$; in fact $a^{\prime}+a+a^{\prime \prime} \subseteq P_{1}$ and $a^{\prime} \in a^{\prime}+e^{\prime \prime} \subseteq a^{\prime}+a+a^{\prime \prime}$, also $a^{\prime \prime} \in e^{\prime}+a^{\prime \prime} \subseteq a^{\prime}+a+a^{\prime \prime}$.

Now, we prove that $B$ is a complete part of $M$. Let $a \in \sum_{i=1}^{n} m_{i}^{\prime} \cap B \neq \emptyset$, hence a $\sum_{i=1}^{t} z_{i}^{\prime}=P \in T$ exists such that $a \in P$. Now let $e^{\prime}, e^{\prime \prime}$ be respectively the left and right identities, $a^{\prime}, a^{\prime \prime} \in M$, such that $e^{\prime} \in a^{\prime}+a, e^{\prime \prime} \in a+a^{\prime \prime}$. Then $\sum_{i=1}^{n} m_{i}^{\prime} \subseteq e^{\prime}+\sum_{i=1}^{n} m_{i}^{\prime}+e^{\prime \prime} \subseteq a^{\prime}+a+\sum_{i=1}^{n} m_{i}^{\prime}+a+a^{\prime \prime} \subseteq$ $a^{\prime}+P+\sum_{i=1}^{n} m_{i}^{\prime}+P+a^{\prime \prime} \supseteq a^{\prime}+a+a+a^{\prime \prime} \supseteq\left\{e^{\prime}, e^{\prime \prime}\right\}$, thus $a^{\prime}+P+$ $\sum_{i=1}^{n} m_{i}^{\prime}+P+a^{\prime \prime}=P_{1}$. Therefore $\sum_{i=1}^{n} m_{i}^{\prime} \subseteq P_{1} \in T$ and for this reason
$\sum_{i=1}^{n} m_{i}^{\prime} \subseteq B$.

Let $a, b \in M$, such that $a \in P, b \in Q$ where $P, Q \in T$. Then $a+b \in B$. Also for every $r \in R, r \cdot a \subseteq B$.

Also, $B$ satisfies the conditions of reproducibility. Since $M$ is an $R$-hypermodule, the properties of $M$ as an $R$-hypermodule, guarantee that the hypergroup $B$ is an $R$-hypermodule. It is clear that $B \subseteq \omega(M)$. As seen from the above, it turns out that $B$ is a complete part subhypermodule, thus by Lemma $2.4, \omega(M) \subseteq B$.

We denote by $\sum_{C}(A)$ the set of hypersums $A$ of elements of $M$ such that $C(A)=A$.

Theorem 2.6. Let $M$ be an R-hypermodule and $\left(x_{1}^{\prime}, \ldots, x_{n}^{\prime}\right)$ such that $\sum_{i=1}^{n} x_{i}^{\prime} \in \sum_{C}(M)$, then there exists $\left(y_{1}^{\prime}, \ldots, y_{n}^{\prime}\right)$ such that $\sum_{i=1}^{n} x_{i}^{\prime}+$
$\sum_{i=1}^{n} y_{i}^{\prime}=\omega(M)$. 
Proof. We set $x_{i}^{\prime}=\sum_{j=1}^{n_{i}}\left(\prod_{k=1}^{k_{i j}} r_{i j k}\right) x_{i}$. For $1 \leq t \leq n$, let $a_{t}$ be an element of $\omega(M)$, then there exists $y_{t} \in M$ such that $a_{t} \in x_{t}+y_{t}$, hence

$$
\sum_{j=1}^{n_{t}}\left(\prod_{k=1}^{k_{t j}} r_{t j k}\right) a_{t} \subseteq \sum_{j=1}^{n_{t}}\left(\prod_{k=1}^{k_{t j}} r_{t j k}\right) x_{t}+\sum_{j=1}^{n_{t}}\left(\prod_{k=1}^{k_{t j}} r_{t j k}\right) y_{t}=x_{t}^{\prime}+y_{t}^{\prime} .
$$

Since $\omega(M)$ is a complete part, we have $x_{t}^{\prime}+y_{t}^{\prime} \subseteq \omega(M)$. Therefore

$$
\begin{aligned}
\sum_{i=1}^{n} x_{i}^{\prime}+y_{n}^{\prime} & =\omega(M)+\sum_{i=1}^{n} x_{i}^{\prime}+y_{n}^{\prime}=\sum_{i=1}^{n-1} x_{i}^{\prime}+\omega(M)+x_{n}^{\prime}+y_{n}^{\prime} \\
& =\sum_{i=1}^{n-1} x_{i}^{\prime}+\omega(M)=\omega(M)+\sum_{i=1}^{n-1} x_{i}^{\prime}
\end{aligned}
$$

and so

$$
\sum_{i=1}^{n} x_{i}^{\prime}+y_{n}^{\prime}+y_{n-1}^{\prime}=\omega(M)+\sum_{i=1}^{n-2} x_{i}^{\prime}+x_{n-1}^{\prime}+y_{n-1}^{\prime}=\omega(M)+\sum_{i=1}^{n-2} x_{i}^{\prime} .
$$

Going on the same way one arrives to

$$
\sum_{i=1}^{n} x_{i}^{\prime}+\sum_{i=1}^{n} y_{i}^{\prime}=\omega(M)+x_{1}^{\prime}+y_{1}^{\prime}=\omega(M)
$$

LemMA 2.7. Let $(M,+)$ be an R-hypermodule, then

(1) $M-\omega(M)$ is a complete part of $M$.

(2) If $M-\omega(M)$ is a hypersum, then $\omega(M)$ is also a hypersum.

Proof. (1) Obvious.

(2) By (1), $M-\omega(M)$ is a complete part. Now, by using Theorem 2.6, the proof is completed.

REMARK 2.8. Let $M$ be an $R$-hypermodule endowed with a complete hypersum. The following implication is satisfied for every $A \in \wp^{*}(M)$ : $A \cap \sum_{i=1}^{n} m_{i}^{\prime}=\emptyset \Rightarrow C(A) \cap \sum_{i=1}^{n} m_{i}^{\prime}=\emptyset$.

Suppose that $z \in C(A) \cap \sum_{i=1}^{n} m_{i}^{\prime}$. Then $a \in A$ exists such that $z \in C(a)$, hence $C(a)=C(z)$. The hypothesis $\sum_{i=1}^{n} m_{i}^{\prime}=C\left(\sum_{i=1}^{n} m_{i}^{\prime}\right)$ implies

$$
C(z) \subseteq \bigcup_{y \in \sum_{i=1}^{n} m_{i}^{\prime}} C(y)=C\left(\sum_{i=1}^{n} m_{i}^{\prime}\right)=\sum_{i=1}^{n} m_{i}^{\prime}
$$


Therefore $a \in A, a \in C(z) \subseteq \sum_{i=1}^{n} m_{i}^{\prime}$, where $\sum_{i=1}^{n} m_{i}^{\prime} \cap A \neq \emptyset$ which is absurd.

Let $(M,+)$ be an $R$-hypermodule. Let's consider the sequence

(*) $\quad M \supseteq \omega(M)=\omega_{1} \supseteq \omega(\omega(M))=\omega_{2} \supseteq$

$$
\cdots \supseteq \omega_{k} \supseteq \omega_{k+1} \supseteq \cdots \supseteq \omega_{n} \supseteq \cdots
$$

Proposition 2.9. Let $M$ be an R-hypermodule. Then the following conditions are equivalent:

(1) The sequence (*) is finite;

(2) there is $(n, k) \in \mathrm{N}^{2}$, where $n>k+1$, such that $\omega_{n}$ is a complete part of $\omega_{k}$;

(3) there is $(n, k) \in \mathrm{N}^{2}$ where $n>k+1$, such that for any $(x, y) \in$ $\left(\omega_{k}-\omega_{n}\right) \times\left(\omega_{k}-\omega_{n}\right) ;(x+y) \cap\left(\omega_{k}-\omega_{n}\right) \neq \emptyset$ implies $x+y \subseteq \omega_{k}-\omega_{n} ;$

(4) there is $(n, k) \in \mathrm{N}^{2}$, where $n>k+1$, such that for any $\omega_{n}$ is a $\omega_{k}$ conjugable.

Proof. (1) $\Rightarrow(2)$. If the sequence $(*)$ is finite, then there is $n \in \mathrm{N}$ such that $\omega_{n}=\omega_{n-1}$, hence $\omega_{n}$ is a complete part of $\omega_{n-2}$.

(2) $\Rightarrow(3)$. If $\omega_{n}$ is a complete part of $\omega_{k}$, then $\omega_{k}-\omega_{n}$ is a complete part of $\omega_{k}$.

$(3) \Rightarrow(4)$. One proves easily that for any $s \in \mathrm{N}, \omega_{s}$ is a closed subhypermodule of $M$. Moreover, for all $a, b \in \omega_{k}$, if $\{a, b\} \subseteq \omega_{k}-\omega_{n}$, we have $a+b \subseteq \omega_{k}$, if $a \neq b$ and $\left|\{a, b\} \cap \omega_{n}\right|=1$, we have $a+b \subseteq \omega_{k}-\omega_{n}$, since $\omega_{n}$ is a closed subhypermodule of $\omega_{k}$. Then, we obtain that $\omega_{n}$ is $\omega_{k}$-conjugable.

(4) $\Rightarrow(1)$. We know $\omega_{n}$ is a complete part subhypermodule of $\omega_{k}$. Hence $\omega_{k+1}=\omega\left(\omega_{k}\right) \subseteq \omega_{n} \subseteq \omega_{k+1}$ from which $\omega_{n}=\omega_{k+1}$. So, we have: $\omega_{n+1}=$ $\omega\left(\omega_{n}\right)=\omega\left(\omega_{k+1}\right)=\omega_{k+2} \supseteq \omega_{n}=\omega_{k+1} \supseteq \omega_{k+2}$. Therefore, $\omega_{n}=\omega_{k+2}=$ $\omega_{n+1}$. Let $\omega_{n+s}=\omega_{k+1}$. It follows $\omega_{n+s+1}=\omega\left(\omega_{n+s}\right)=\omega\left(\omega_{k+1}\right)=\omega_{k+2}=$ $\omega_{k+1}$. Then, for any $m$ such that $m \geq n$, we have $\omega_{m}=\omega_{n}$.

THEOREM 2.10. Let $(M,+)$ be an $R$-hypermodule such that the sequence (*) is finite, and let $N$ be a complete part subhypermodule of $M$. Then there is $p \in \mathrm{N}$ such that $\omega_{p+1}(N)=\omega_{p+1}(M)$.

Proof. Notice that $\omega(N)$ is a subhypermodule of $\omega(M)$. Indeed, for any $a \in \omega(N)$, there is $e \in N$ such that $a \in a+e$, it's clear that $a \in \epsilon_{N}(e) \subseteq$ $\epsilon_{M}(e)=\omega(M)$. Moreover, since $N$ is a complete part subhypermodule of $M$, we have $\omega(M) \subseteq N$. Then $\omega_{1}(N) \subseteq \omega_{1}(M) \subseteq N$. For any $s \geq 1$, from $\omega_{s}(N) \subseteq \omega_{s}(M) \subseteq \omega_{s-1}(N)$, one obtains $\omega_{s+1}(N) \subseteq \omega_{s+1}(M) \subseteq \omega_{s}(N)$, hence a sequence $N \supseteq \omega_{1}(M) \supseteq \omega_{1}(N) \supseteq \omega_{2}(M) \supseteq \omega_{2}(N) \supseteq \cdots$. 
By Proposition 2.9, there is $(n, p) \in \mathrm{N} \times \mathrm{N}$, where $n>p+1$, such that $\omega_{n}(M)=\omega_{p+1}(M)$, therefore $\omega_{p+1}(M)=\omega_{p+1}(N)$.

REMARK 2.11. If $N_{1}, N_{2}$ be subhypermodules of $M$, then

$$
\omega\left(N_{1} \cap N_{2}\right) \leq \omega\left(N_{1}\right) \cap \omega\left(N_{2}\right) .
$$

Generally, we do not have equality.

Let $M$ be an $R$-hypermodule, for which $\omega(M) \neq M$ and let $x, y \in M$ be arbitrary in $M$. Let's define on $M^{\prime}=M \cup\{b, c, d\}(\{b, c, d\} \cap M=\emptyset)$ the following hyperoperation:

\begin{tabular}{c|cccc}
$+^{\prime}$ & $x$ & $b$ & $c$ & $d$ \\
\hline$y$ & $y+x$ & $b$ & $c$ & $d$ \\
$b$ & $b$ & $M$ & $d$ & $c$ \\
$c$ & $c$ & $d$ & $M$ & $b$ \\
$d$ & $d$ & $c$ & $b$ & $M$
\end{tabular}

and for every $r \in R, m \in M$ the scalar multiplication $r \cdot^{\prime} m=r \cdot m$ and $r{ }^{\prime} a=a, r \cdot^{\prime} b=b$ and $r \cdot^{\prime} c=c$. We can easily verify $\left(M^{\prime},+\right)$ with scalar multiplication ' ${ }^{\prime}$ is an $R$-hypermodule. We consider subhypermodules $M_{1}^{\prime}=M \cup\{b\}, M_{2}^{\prime}=M \cup\{c\}, M_{3}^{\prime}=M \cup\{d\}$ of $M^{\prime}$, then $\omega\left(M_{1}^{\prime}\right)=\omega\left(M_{2}^{\prime}\right)=$ $\omega\left(M_{3}^{\prime}\right)=M, \omega\left(M_{1}^{\prime} \cap M_{2}^{\prime} \cap M_{3}^{\prime}\right) \neq M$.

But for an $R$-hypermodule $M$, and $N_{1}, N_{2} \leq M$ whose sequence (*) is finite, we can find the following relation between $\omega\left(N_{1} \cap N_{2}\right)$ and $\omega\left(N_{1}\right), \omega\left(N_{2}\right)$ :

Proposition 2.12. If $N_{1}, N_{2} \leq M$, where $M$ has a finite sequence (*), then there exist $p \in \mathrm{N}$, such that $\omega_{p+1}\left(N_{1} \cap N_{2}\right)=\omega_{p+1}\left(\omega\left(N_{1}\right) \cap \omega\left(N_{2}\right)\right)$.

Proof. Let's consider $\bar{M}:=N_{1} \cap N_{2}$ and $\bar{N}:=\omega\left(N_{1}\right) \cap \omega\left(N_{2}\right)$. Then $\bar{N}$ is a subhypermodule, complete part of $\bar{M}$. (We can verify this using the definition of a complete part of a hypermodule.) Therefore we use the proof of Theorem 2.10.

Also, we can give a relation for $R$-subhypermodule of $M$ :

$\exists p \in \mathrm{N}, \quad \omega_{p+1}\left(N_{1} \cap N_{2} \cap \cdots \cap N_{m}\right)=\omega_{p+1}\left(\omega\left(N_{1}\right) \cap \omega\left(N_{2}\right) \cap \cdots \cap \omega\left(N_{m}\right)\right)$.

REMARK 2.13. If $N_{1}, N_{2} \leq M$, then $\omega\left(N_{1}\right) \subseteq N_{1} \cap \omega\left(\left\langle N_{1} \cup N_{2}\right\rangle\right)$.

Generally, we have not equality. Let $M_{1}$ and $M_{2}$ be two $R$-hypermodules with the scalar hyperoperation $\cdot_{1}$ and.$_{2}$ respectively. Let $m_{1}, n_{1}$ arbitrary in $M_{1}$ 
and $m_{2}, n_{2}$ arbitrary in $M_{2}$. Let's define on $M=M_{1} \cup M_{2} \cup\{a\}\left(a \notin M_{1} \cup M_{2}\right)$ with the following hyperoperations:

\begin{tabular}{c|ccc}
$+^{\prime}$ & $m_{1}$ & $a$ & $m_{2}$ \\
\hline$n_{1}$ & $n_{1}+m_{1}$ & $a$ & $M$ \\
$a$ & $a$ & $M_{1}$ & $M$ \\
$n_{2}$ & $M$ & $M$ & $n_{2}+m_{2}$
\end{tabular}

and for every $r \in R, x \in M_{1}$ and $y \in M_{2}$ the scalar multiplication $r \cdot^{\prime} x=r \cdot{ }_{1} x$, $r \bullet^{\prime} y=r \cdot 2 y$ and $r \cdot^{\prime} a=a$. We can easily verify $\left(M,+^{\prime}\right)$ with scalar multiplication ${ }^{\prime}$ is an $R$-hypermodule. We consider subhypermodules $N_{1}=$ $M_{1} \cup\{a\}, N_{2}=M_{2}, N_{1} \cup N_{2}=M,\left\langle N_{1} \cup N_{2}\right\rangle=M$, then $\omega\left(\left\langle N_{1} \cup N_{2}\right\rangle\right)=M$. So

$$
\omega\left(N_{1}\right)=M_{1} \subset N_{1} \cap \omega\left(\left\langle N_{1} \cup N_{2}\right\rangle\right)=N_{1}=M_{1} \cup\{a\} .
$$

THEOREM 2.14. Let $M$ be an R-hypermodule with commutative hypergroup and $N_{1}, N_{2}$ be subhypermodules of $M$. If for any $a \in\left\langle N_{1} \cup N_{2}\right\rangle-\left(N_{1} \cup N_{2}\right)$, there exists $\left(n_{1}, n_{2}\right) \in N_{1} \times N_{2}$, such that $a \in n_{1}+n_{2}$ and if $\left\langle\omega\left(N_{1}\right) \cup \omega\left(N_{2}\right)\right\rangle$ is a closed subhypermodule of $\omega\left(\left\langle N_{1} \cup N_{2}\right\rangle\right)$ then

$$
\left\langle\omega\left(N_{1}\right) \cup \omega\left(N_{2}\right)\right\rangle=\omega\left(\left\langle N_{1} \cup N_{2}\right\rangle\right) .
$$

Proof. We shall prove that $\left\langle\omega\left(N_{1}\right) \cup \omega\left(N_{2}\right)\right\rangle$ is conjugable in $\left\langle N_{1} \cup N_{2}\right\rangle$ as hypermodule. $\left\langle\omega\left(N_{1}\right) \cup \omega\left(N_{2}\right)\right\rangle$ is closed in $\left\langle N_{1} \cup N_{2}\right\rangle$ because, from $x \in$ $a+b$, where $(a, b) \in\left\langle\omega\left(N_{1}\right) \cup \omega\left(N_{2}\right)\right\rangle^{2}$ and $x \in\left\langle N_{1} \cup N_{2}\right\rangle$, it results $(a, b) \in \omega\left(\left\langle N_{1} \cup N_{2}\right\rangle\right)^{2}$ and so $x \in \omega\left(\left\langle N_{1} \cup N_{2}\right\rangle\right)$. Using now the condition given in the proposition, $x \in\left\langle\omega\left(N_{1}\right) \cup \omega\left(N_{2}\right)\right\rangle$.

As regards an arbitrary element $a \in\left\langle N_{1} \cup N_{2}\right\rangle$, we have three cases:

$$
\begin{gathered}
a \in N_{1} \Rightarrow \exists a^{\prime} \in N_{1}, a+a^{\prime} \subseteq \omega\left(N_{1}\right) \subseteq\left\langle\omega\left(N_{1}\right) \cup \omega\left(N_{2}\right)\right\rangle ; \\
a \in N_{2} \Rightarrow \exists a^{\prime} \in N_{2}, a+a^{\prime} \subseteq \omega\left(N_{2}\right) \subseteq\left\langle\omega\left(N_{1}\right) \cup \omega\left(N_{2}\right)\right\rangle ; \\
a \in\left\langle N_{1} \cup N_{2}\right\rangle-\left(N_{1} \cup N_{2}\right) \Rightarrow \exists n_{1} \in N_{1}, \exists n_{2} \in N_{2}, a \in n_{1}+n_{2} .
\end{gathered}
$$

For $n_{i}$ there exists $n_{i}^{\prime} \in N_{i}$, such that $n_{i}+n_{i}^{\prime} \in \omega_{n_{i}}, i=1,2$.

So, $a+n_{1}^{\prime}+n_{2}^{\prime} \subseteq\left(n_{1}+n_{2}^{\prime}\right)+\left(n_{2}+n_{2}^{\prime}\right) \subseteq \omega\left(N_{1}\right) \oplus \omega\left(N_{2}\right) \subseteq<\omega\left(N_{1}\right) \cup$ $\omega\left(N_{2}\right)>$, whence for every $t \in n_{1}^{\prime}+n_{2}^{\prime}, a+t \subseteq<\omega\left(N_{1}\right) \cup \omega\left(N_{2}\right)>$.

A hypermodule $M$ is said to be $\epsilon_{n}^{*}$-complete hypermodule if there exists $n \in \mathrm{N} \cup\{0\}$, and $n$ is the smallest integer such that $\epsilon_{n}^{*}=\epsilon^{*}$ and $\epsilon_{n}^{*} \neq \epsilon_{n-1}^{*}$.

LemMa 2.15. A hypermodule $M$ is $\epsilon_{0}^{*}$-complete if and only if $M$ is a hypermodule (with ordinary group). 
Proof. Suppose that $M$ is a $\epsilon_{0}^{*}$-complete hypermodule, so $\epsilon_{0}^{*}=\epsilon^{*}$, hence $\epsilon_{2} \subseteq \epsilon_{0}$ and $\epsilon_{1} \subseteq \epsilon_{0}$. Now, for every $x, y \in m_{1}+m_{2}$, we have $x \epsilon_{2} y$, so $x=y$. Also for every $x, y \in r . m$, we have $x \epsilon_{1} y$, so $x=y$. Therefore $m_{1}+m_{2}$ and $r \cdot m$ both are singletons, and so $M$ is a hypermodule over the hyperring $R$.

Conversely, if $M$ is a module, then for every $\sum_{i=1}^{n} m_{i}^{\prime}$, we have $\left|\sum_{i=1}^{n} m_{i}^{\prime}\right|=$ 1. By definition, $x \epsilon_{n} y$ if and only if $x=\sum_{i=1}^{n} m_{i}^{\prime}=y$, thus $x=y$ and $x \epsilon_{0} y$.

Corollary 2.16. If $M$ is a $\epsilon_{n}^{*}$-complete $R$-hypermodule, then $M / \epsilon_{n}^{*}$ is an $R / \Gamma^{*}$-module.

Proposition 2.17. Every finite hypermodule is $\epsilon_{n}^{*}$-complete.

Proof. Since $M$ is finite, the chain $\epsilon_{1}^{*} \subseteq \epsilon_{2}^{*} \subseteq \cdots$ is stationary. Thus there exists $n \in \mathrm{N}$ such that $\epsilon_{n}^{*}=\epsilon^{*}$ and $\epsilon_{n}^{*} \neq \epsilon_{n-1}^{*}$.

TheOREM 2.18. We have

(1) If $\forall(v, w) \in(\omega(M))^{2}, v \epsilon_{n} w$, then $\epsilon=\epsilon_{n+1}$.

(2) If $\forall(v, w) \in(\omega(M))^{2}, v \epsilon_{n}^{*} w$, then $\epsilon^{*}=\epsilon_{n+1}^{*}$.

Proof. (1) If $x \epsilon y$, since $\omega(M)+M=M+\omega(M)=M$ then there exists $(v, w) \in(\omega(M))^{2}$ such that $y \in x+v$ and $y \in x+w$, by the hypothesis $v \epsilon_{n} w$. Now, we have $(x+v) \overline{\bar{\epsilon}}_{n+1}(x+w)$, whence $x \epsilon_{n+1} y$, so $\epsilon \subseteq \epsilon_{n+1}$.

(2) It follows from (1).

Let $M$ be an $R$-hypermodule. $M$ is called 1-hypermodule if $\omega(M)$ is a singleton.

THEOREM 2.19. Let $M$ be an 1-hypermodule and $\omega(M)=\{e\}$. Then

(1) The $\epsilon^{*}$-classes are the summations $e+a$, where $a \in M$.

(2) Every $R$-subhypermodule of $M$ is complete part.

(3) If $\left\{M_{i}\right\}_{i \in I}$ is a family of $R$-subhypermodules of $M$, then $\bigcap_{i \in I} M_{i}$ is an $R$-subhypermodule of $M$.

(4) The direct product of 1-hypermodules is a 1-hypermodule.

Proof. (1) It is clear.

(2) If $N$ is a subhypermodule of $M$, we have $N \cap \omega(M) \neq \emptyset$, which implies $\omega(M) \subseteq N$, hence $N=N+\omega(M)$ and therefore $N$ is a complete part.

(3) For (2), $\forall i \in I, e \in M_{i}$, we set $N=\bigcap_{i \in I} M_{i}$, hence $N \neq \emptyset$. Then for every $x, y \in N$ there exists $b \in M$ such that $y \in b+x$, but $M_{i}$ is $(\forall i \in I)$ a closed submodule by (2), thus $b \in M_{i}$. Also, for every $r \in R, m \in N$, we have $r \cdot m \subseteq N$.

(4) Set $N=\prod_{i \in I} N_{i}, x^{\prime}=\left(x_{i}^{\prime}\right)_{i \in I} \in N, e=\left(e_{i}\right)_{i \in I}$. We have $x \epsilon_{n} e$ if and only if $z^{\prime 1}=\left(z_{i}^{\prime 1}\right)_{i \in I}, z^{\prime 2}=\left(z_{i}^{\prime 2}\right)_{i \in I}, \ldots, z^{\prime n}=\left(z_{i}^{\prime n}\right)_{i \in I}$ exist such 
that $x, e \in \sum_{i=1}^{n} z^{\prime k}$, that is if and only if $\forall i \in I, x_{i}^{\prime}, e_{i} \in \sum_{k=1}^{n} z_{i}^{\prime k}$. Then $z_{i}^{\prime}=\sum_{k=1}^{n} z_{i}^{\prime k}=e_{i}$, whence $x=e$, for this reason $\omega(N)=\{e\}$.

ACKNOWLEDGEMENT. The authors are highly grateful to referees for their valuable comments and suggestions for improving the paper.

\title{
REFERENCES
}

1. Anvariyeh, S. M., and Davvaz, B., $\theta$-Closure and $\theta$-parts of hypermodules, Algebra Colloq (to appear).

2. Corsini, P., Prolegomena of Hypergroup Theory, Aviani Editore, Tricesimo 1993.

3. Corsini, P., and Leoreanu, V., Applications of Hyperstructure Theory, Advances in Math. 5, Kluwer, Dordrecht 2003.

4. Davvaz, B., Remark on weak hypermodules, Bull. Korean Math. Soc. 36 (1999), 599-608.

5. Davvaz, B., and Vougiouklis, T., Commutative rings obtained from hyperrings ( $H_{v}$-rings) with $\alpha^{*}$-relations, Comm. Algebra 35 (2007), 3307-3320.

6. Vougiouklis, T., The fundamental relation in hyperrings. The general hyperfield, pp. 203211 in: Algebraic Hyperstructures and Applications, Proc. Xánthi 1990, World Scientific, Teaneck, NJ 1991.

7. Vougiouklis, T., Hyperstructures and their Representations, Hadronic Press, Palm Harber, FL 1994.

\author{
DEPARTMENT OF MATHEMATICS \\ YAZD UNIVERSITY \\ YAZD \\ IRAN \\ E-mail:davvaz@yazduni.ac.ir
}

